In: Galdynski; Kühl (Hrsg.), Black-Box Beratung? Empirische Studien zu Coaching und Supervision, Wiesbaden 2009: VS Verlag für Sozialwissenschaften: 47-69.

\title{
Die nur fast gelingende Schließung des Personalentwicklungszyklus
}

\author{
Stefan Kühl
}

\section{Wie kommt es zur Popularität von Personalentwicklungsmaßnahmen?}

Weswegen werden in Unternehmen, Verwaltungen, Krankenhäusern und anderen Organisationen zunehmend Personalentwicklungsmaßnahmen wie Trainings, Coaching oder Supervision nachgefragt? Lassen sich Supervision und Coaching als Reaktionen auf diese Entwicklungen verstehen, wie sie durch soziologische Erklärungsansätze von der Subjektivierung der Arbeit (vgl. Moldaschl 2005), der Ausbildung von Arbeitskraftunternehmern (vgl. Voß, Pongratz 1998) oder von der Entberuflichung (vgl. Beck 1986) nahe gelegt werden? Verändern sich die Mechanismen, mit denen Organisationen ihre Mitglieder inkludieren, und ist die Ausbildung von personenorientierter Beratung in Organisationen eine Reaktion darauf?

Bei der Begründung für den allgemein konstatierten wachsenden Bedarf stößt man bei den Selbstbeschreibungen der Personalentwickler in der Regel auf die „üblichen Verdächtigen“, die für Neuausrichtungen von Organisationen verantwortlich gemacht werden: Globalisierung, Internationalisierung der Organisationen, verstärkter Einsatz von Informations- und Kommunikationstechnologien, Wettbewerbsdruck oder die Virtualisierung der Arbeitsbeziehungen (vgl. z.B. Buchinger 2002, S. 49; Thommen 2005, S. 64). Tätigkeiten in Organisationen, so die Standardargumentation, werden immer weniger regelhaft. Viele Arbeitsvollzüge, so z.B. Belardi (2002, S. 11), würden immer komplizierter und deswegen tendenziell zu einer Überforderung von Mitarbeitern, aber auch ganz besonders von Führungskräften führen. In vielen Bereichen lösten sich, so Reichel \& Rabenstein (2001, S. 10), die Gewissheiten auf, „wie etwas klarerweise zu tun und zu werten ist." Wenn es dabei auch noch um die "Arbeit mit Menschen“ gehe, 
dann komme noch die Unklarheit hinzu, „was wie wirksam ist" und „was man als erfolgreiche Arbeit bezeichnen kann."

In der Regel wird in der Literatur über Supervision und Coaching mit einer vierstufigen Argumentationskette gearbeitet, um die Ausdifferenzierung der personenorientierten Beratung in Organisationen zu erklären (vgl. Buchinger 2002, S. 49): 1. Die Kontextbedingungen von Organisationen in der Wirtschaft, der Politik, der Bildung und des Gesundheits- und Sozialwesens hätten sich verändert. 2. Dadurch bildeten sich neue Erwartungen an Selbstkontrolle, Selbstökonomisierung und Selbstrationalisierung der Beschäftigten in Organisationen. 3. Die verstärkte selbstständige Planung, Steuerung und Überwachung der eigenen Tätigkeiten, die zunehmend geforderte „Produktion“ und „Vermarktung“ der eigenen Fähigkeiten und Leistungen und die mit der Auflösung der Trennung von Berufs- und Privatleben einhergehende „Verbetrieblichung der Lebensführung" stellten dann neue, nur schwer zu bewältigende Anforderungen an die Beschäftigten dar, weil sich nicht nur ihre Handlungs- und Gestaltungsspielräume erhöhten, sondern auch der Leistungsdruck stark zunehme (vgl. Pongratz 2004, S. 20). 4. Dadurch entstehe ein "neuartiger Bedarf“ an personenorientierter Beratung, der durch die Angebote von Supervisoren und Coachs befriedigt werde (vgl. Siller/Buer 2004, S. 10).

Dieser Erklärungsansatz liegt aus einer arbeitssoziologischen, aber übrigens auch aus einer betriebswirtschaftlichen und arbeitspsychologischen Forschungsperspektive nahe. Die Fremdbeschreibungen der Wissenschaftler überschneiden sich hier mit den Selbstbeschreibungen der Supervisoren und Coachs. Wissenschaftler können deswegen fast eins zu eins Interviewsequenzen mit personenorientierten Beratern, mit Personalentwicklern oder mit Klienten in Organisationen übernehmen, um ihre Argumentation zu belegen. Ihre Schlussfolgerung ist anschlussfähig an die Praktiker, kann sie doch den wachsenden Bedarf nach personenorientierter Beratung wissenschaftlich begründen.

Diese Argumentation ist - gerade aus den oben erwähnten Perspektiven plausibel, sie bringt jedoch einige Verkürzungen mit sich. Es ist fraglich, ob sich Prozesse in einer gern als ",komplex“, "reflexiv“ oder "postmodern“ bezeichneten Gesellschaft kausal aus Umweltbedingungen ableiten lassen. Der Kontingenzansatz, der dies in den 1960er und 1970er Jahren für die Ausbildung von Organisationstypen versucht hat, hat stark an Plausibilität eingebüßt (vgl. Kieser 1995). Ohne noch explizit auf ihn zurückzugreifen, schimmert er bei der Erklärung der Ausbildung von neuartigen Beratungsansätzen aber mehr als deutlich durch. Coaching, Supervision oder Lernprojekte werden als die Lösungen angeboten, mit denen die Mitarbeiter in Unternehmen, Verwaltungen, Verbänden und ande- 
ren Organisationen in die Lage versetzt werden, mit den gestiegenen Anforderungen zurechtzukommen. Das Muster ist das eines einfachen Problem-LösungsSchemas: Die Mitarbeiter in den Organisationen haben Probleme, und die Personalentwicklung liefert die entsprechenden Qualifikationspakete, damit die Mitarbeiter diese Probleme bewältigen können.

Es ist aus einer soziologischen Perspektive überraschend, dass gerade in der Disziplin der Personalentwickler, in der gerne von der "systemischen Unterscheidung von Berater- und Klientensystem“, der "Unterscheidung von Selbst- und Fremdreferenz" oder gar von der "Autopoiesis sozialer Systeme" gesprochen wird (vgl. z.B. König/Vollmer 2002; Backhausen/Thommen 2004), die Nachfrage nach den eigenen Dienstleistungen mit einem einfachen Problem-Lösungs-Mechanismus erklärt wird. Gibt es hier einen blinden Fleck, wenn systemisch geprägte Personalentwickler, Trainer oder Coachs über ihre eigene Disziplin reden?

In diesem Artikel wird die gewachsene Nachfrage nach Personalentwicklungsmaßnahmen nicht allein mit den veränderten Umweltanforderungen an Organisationen erklärt, sondern mit Problemlagen, die sich die Organisationen selbst geschaffen haben. Die These ist, dass die Nachfrage besonders nach neuartigen Personalentwicklungsmaßnahmen vorrangig durch den Boom der Personaldiagnostikmaßnahmen in den letzten zwanzig Jahren entstanden ist. Erst durch Maßnahmen wie die Potenzialanalyse, Führungskräfte-Assessment-Center, 360-Grad-Feedback konnten einige Trainingsmaßnahmen, aber besonders die personenbezogenen Beratungen durch Coachs oder Supervisoren, an Attraktivität gewinnen. Es bilden sich, so das Argument, in Organisationen zunehmend Personalentwicklungszyklen aus - eine Art personaler "Rundumversorgung" von Mitarbeitern mit Instrumenten der Personaldiagnostik, der Personalentwicklungsmaßnahmen und der Fortschrittsmessung.

Die hier entwickelte These basiert auf Untersuchungen in 18 Organisationen, die im Sommer 2005 durchgeführt wurden. Etwas mehr als 60\% der Organisationen sind Unternehmen. In diesem Organisationstyp wurde die am weitesten fortgeschrittene Verankerung von modernen Personalentwicklungsinstrumenten wie Coaching vermutet. Die restlichen Organisationen kamen aus den Bereichen Kirche, Verwaltung, Gewerkschaft und Sozialhilfe. Die Personalentwicklungsstrategie der 18 Organisationen wurde durch Interviews, Dokumentenanalyse und Analyse der Websites rekonstruiert. Im Mittelpunkt standen dabei die zwischen einer und drei Stunden langen leitfadengestützten Experteninterviews mit den Personalentwicklern. Die Personalentwickler wurden von uns deswegen als Gesprächspartner ausgewählt, weil sie in Organisationen die Positionen innehaben, in denen maßgeblich über den Einsatz von Personaldiagnostik- und Inter- 
ventionsinstrumenten innerhalb der Organisation entschieden wird. Wegen der Scharnierfunktion zwischen Anbieter und Klient kann diese Untersuchung als eine Gatekeeper-Studie betrachtet werden.

Die von uns verwandte Methode der Experteninterviews eignet sich unseres Erachtens besonders gut zur Rekonstruktion eines noch nicht systematisch erforschten Feldes (zur Methodik siehe Liebold/Trinczek 2002). Die Methode des Experteninterviews wurde auch deswegen gewählt, weil es uns nicht nur um eine Abfrage von Ersteinschätzungen der Personalentwickler ging (was eine quantitative Herangehensweise oder schriftliche Befragung nahe gelegt hätte), sondern um die möglichst detaillierte Rekonstruktion der Denkstrukturen in der Personalentwicklung. Die Darstellung, Kategorisierung und Interpretation wurde deswegen nicht allein den interviewten Personalentwicklern überlassen. Ziel war es dabei, sich relativ stark von den bewährten Bewertungsmustern der Experten zu entfernen und so differente, teilweise kontrainduktive Ergebnisse herauszukristallisieren (grundsätzlich dazu siehe Strodtholz/Kühl 2002, im Speziellen zu unserem Projekt Taffertshofer 2007). ${ }^{1}$

Im folgenden zweiten Abschnitt wird die Konstruktionsform dieses Personalentwicklungszyklus vorgestellt. Dabei wird gezeigt, wie Personaldiagnostik, Intervention und Fortschrittsevaluierung ineinander greifen. Die Ausbildung dieses Personalentwicklungszyklus führt, so die Argumentation im dritten Abschnitt, zu einer Verschiebung der Machtverhältnisse bei Personalentscheidungen. Die Kompetenzen verlagern sich tendenziell von den Führungskräften auf die Personaler. Dieser Terraingewinn der Personaler in Organisationen ist jedoch problematisch. Die Personalentwickler kommen in eine Reihe neuer Dilemmata, die im vierten Abschnitt näher spezifiziert werden. Im abschließenden fünften Abschnitt wird die Ausbildung dieses Personalentwicklungszyklus in einem größeren Kontext diskutiert. ${ }^{2}$

\footnotetext{
${ }^{1}$ Bis auf zwei Interviews wurden alle Gespräche aufgezeichnet und komplett transkribiert. Bei den beiden nicht aufgezeichneten Gesprächen wurden die Aussagen des Gesprächspartners mitprotokolliert und die dabei wörtlich protokollierten Sätze besonders markiert. Die Anführung von Daten, Positionen und Zitaten erfolgt hier, wie auch in den weiteren Ausarbeitungen, streng anonymisiert, das heißt in einer Form, in der weder Personen noch Organisationen rekonstruiert werden können. Zur Sicherstellung der Anonymität von Organisationen und Interviewpartnern wurden Angaben, die nicht für die Aussage relevant sind (z.B. Branche, Ortsangabe oder Geschlecht der Gesprächspartner) teilweise verändert.

${ }^{2}$ Eine weiter generalisierte Fassung dieses Arguments findet sich in meinem Buch Kühl 2008, in dem verschiedene Aspekte des Coachings und der Supervision aus einer soziologischen Perspektive beschrieben werden. Dieser Beitrag ist in leicht veränderter Form im Jahr 2008 auch als Artikel in der Zeitschrift Organisationsberatung - Supervision - Coaching erschienen.
} 


\section{Der Zyklus: Das Ineinandergreifen von Diagnostik und Intervention}

Mit der Ausbildung neuer personenorientierter Managementdiskurse seit den 1990er Jahren haben sich auch zahlreiche Personaldiagnostikinstrumente in Unternehmen, aber auch in Verwaltungen, Krankenhäusern, Sozial- und Pflegehilfeeinrichtungen und sogar Non-Profit-Organisationen etablieren können (siehe dazu Boltansky/Chiapello 2003, S. 89 ff.). Bei Führungskräfte-Evaluationen, Mitarbeitergesprächen, Management-Audits, Potenzialanalysen, 360-Grad-Feedbacks oder Nachwuchs-Assessment-Centern geht es darum, die Stärken und Schwächen von bereits in der Organisation tätigen Mitarbeitern einzuschätzen. Das Ziel ist dabei nicht vorrangig, die Eignung der Getesteten für ihre momentane Position zu überprüfen, sondern Informationen darüber zu sammeln, ob sie für hierarchisch höher gestellte Posten geeignet sind.

Dabei kommt es in der Regel zu einer irgendwie gearteten Defizitbestimmung: Die Führungskraft mag beim 360-Grad-Feedback überall gute Werte erreichen, aber in der Arbeit mit Organisationsexternen noch Schwächen zeigen. Das Assessment-Center zeigt, dass der Mitarbeiter seiner jetzigen Aufgabe voll und ganz gewachsen ist, aber in vielen Bereichen noch nicht den Anforderungen der nächsthöheren Führungsposition genügt. Beim Management-Audit werden Schwächen im Ausbalancieren von Arbeitsanforderungen und Privatleben festgestellt.

Aus diesen Defizitbestimmungen durch die Personalentwicklung entsteht sehr häufig die Nachfrage nach Personalentwicklungsmaßnahmen, mit denen diese Defizite reduziert werden können. Als Reaktion auf die Defizitwahrnehmungen in der Personaldiagnostik liefert die Personalentwicklung Angebote in "Paketform": Inhouse-Seminare, offene Einschreibungen in Business Schools, Mentoring mit Top-Management, kollegiale Beratung oder Coaching (vgl. beispielhaft Luthans/Peterson 2003; Smither et al. 2003).

Der Personalentwickler einer süddeutschen Bank berichtet z.B., dass die Personalentwicklung lange Zeit nicht ernst genommen wurde. Seitdem aber die Jahresgespräche in den Zielvereinbarungen gefordert würden und eine „bottom up"-Vorgesetztenbeurteilung eingeführt worden sei, habe sich die Nachfrage nach Personalentwicklungsmaßnahmen stark erhöht (Organisation I, Interview 9). Eine ganze Reihe von "Coaching-Prozessen", so die Personalentwicklerin einer anderen Bank, werde aus der "Potenzial-Analyse“ heraus generiert. Zwar würden viele Anfragen nach Coaching aufgrund neuer Führungsaufgaben kommen, aber die Stärken-Schwächen-Analyse, die in einer Art Assessment-Center 
mündet, bringe konkrete „Entwicklungshinweise“ wie zum Beispiel Seminare zum Thema "Kommunikation und Information“, „Konfliktfähigkeit" oder "Durchsetzungsvermögen“ hervor (Organisation H, Interview 8). In einer großen halbstaatlichen Organisation stellt der Personalentwickler fest, dass bei Mitarbeitergesprächen über „Defizite bei der Leistungserbringung“ gesprochen wird. Bei der Frage, wie diese „Defizite ausgeglichen“ werden könnten, kämen dann "ganz unterschiedliche Maßnahmen“ der Personalentwicklung wie z.B. "Coaching“ ins Gespräch (Organisation G; Interview 7).

Es scheint in einer Reihe von Unternehmen, Verwaltungen und halbstaatlichen Organisationen eine enge Korrelation zwischen der Etablierung von Managementdiagnostik und dem zurzeit sehr populären Coaching zu geben. Bis ungefähr zum letzten Jahrhundertwechsel, so ein Personalentwickler eines großen Industriekonzerns, habe es keine Korrelation von Diagnostik und Coaching gegeben. Jetzt deute sich dies aber immer stärker an. Es gebe jetzt häufiger die Situation, dass aus der Orientierungsklausur, in dem das zukünftige Management ausgewählt wird, die Empfehlung komme, „machen Sie doch mal ein Coaching“, "das ist alles noch nicht so ganz rund“. Auch seien in den letzten Jahren häufiger Kunden gekommen, "die durch ein Management-Audit gegangen sind und die Empfehlung bekommen haben, versuchen Sie, sich da und da zu optimieren mit einem Coaching“ (Organisation F, Interview 6).

Bei der sich deutlich abzeichnenden Verknüpfung von Managementdiagnostik und Intervention haben wir es jedoch bisher noch nicht mit einem geschlossenen Prozess zu tun. Es fehlt dabei die Messung des Erfolgs (oder Misserfolgs) der Intervention (siehe hierzu auch Kühl 2008, Kapitel 3). Hier deuten sich aber in vielen Organisationen bereits Entwicklungstendenzen an, weil die Personalentwickler zunehmend gezwungen sind, den Nutzen der eingesetzten Personalentwicklungsinstrumente nachzuweisen. Eine Personalentwicklerin erklärt, dass man über das Führungskräfte-Feedback einschätzen könne, wie erfolgreich eine Maßnahme ist. „Wenn man alle zwei Jahre das Verfahren mitmacht und sich von den Mitarbeitern einschätzen lässt, dann kann man sagen, O. K., zum Zeitpunkt t1 hatte ich eine Einschätzung. Dann vergingen zwei Jahre, wo ich ein Coaching gemacht habe und an den Punkten gearbeitet habe, die mir meine Mannschaft zurückgemeldet hat. Und im Zeitpunkt t2 kann ich wieder messen: Hat es sich denn wirklich gelohnt, hat es sich verändert?" (Organisation H, Interview 8). Die Personalentwicklerin eines großen Logistikunternehmens erklärt, dass man mit dem jährlich durchgeführten 180-Grad-Feedback messen könne, wie sich die Einschätzung der Führungskräfte durch die Mitarbeiter verändert 
habe, nachdem die Führungskräfte eine Coaching-Maßnahme durchlaufen hatten (Organisation B, Interview 2).

\subsection{Der fast geschlossene Zyklus von Personalbeurteilung, Intervention und Evaluierung}

Den kompletten Prozess aus „Diagnostik - Planung von Interventionen - Intervention - Fortschrittsevaluation mit erneuter Diagnostik" bezeichnen wir als Personalentwicklungszyklus. Mit dem Begriff des Zyklus soll beschrieben werden, dass die verschiedenen Diagnostik-, Interventions- und Evaluierungsinstrumente kreisförmig angeordnet werden und die Führungskräfte nach dem Durchlaufen des Zyklus mehr oder minder automatisch wieder in den nächsten Zyklusdurchlauf eingespeist werden. Der Manager gerät, salopp ausgedrückt, in eine ähnliche Zyklussituation wie neuerdings die Spieler der deutschen Fußballnationalmannschaft. Auch diese werden zuerst auf Schwachstellen getestet, dann durch ein vorrangig individualbezogenes Fitnessprogramm geschickt, danach werden die erzielten Fortschritte gemessen und anschließend werden die Spieler evaluiert.

Durch die schrittweise Etablierung des Personalentwicklungszyklus verändert sich der Status der Personalentwicklung. Die Führungskräfte fragen die Leistungen der Personalentwicklung nicht mehr nur punktuell nach - hier einmal eine bisschen Diagnostik, da mal ein bisschen Coaching -, sondern die Führungskräfte werden in der fortgeschrittenen Variante in ein systematisches Betreuungsverhältnis durch die Personalentwicklung aufgenommen. Personalentwicklung ist aus dieser Perspektive dann nicht mehr ein Leistungsanbieter, bei dem sich eine Führungskraft in einer Art „Boxenstopp“ kurzfristig Hilfestellungen holt, sondern es gibt eine Rundumbegleitung der Führungskraft während ihrer Tätigkeit in einer Organisation.

Der Personalentwicklungszyklus kann idealtypisch als ein Ablauf aus Diagnostik, Maßnahmenplanung, Intervention und Evaluation dargestellt werden; häufig greifen die einzelnen Schritte jedoch nicht in dieser idealtypischen Systematik ineinander. So wird Coaching nicht selten in Anspruch genommen, um sich auf ein Führungskräfte-Assessment-Center vorzubereiten (vgl. Organisation $\mathrm{L}$, Interview 12). Oder die zufällige Teilnahme an einer Trainingsmaßnahme kann dazu führen, dass überhaupt erst ein Defizit wahrgenommen wird, über das die Führungskraft dann durch ein 360-Grad-Feedback Klarheit zu bekommen versucht. 


\subsection{Das Bild des Zyklus in Gewährleistungseinheiten der Organisationen}

Das Konzept des Zyklus bildet sich in verschiedenen Einheiten der Organisation aus. Besonders prominent ist der Shewhart-Deming-Kreis im Qualitätsmanagement. Dieser bereits von Walter Shewhart in den späten 1930er Jahren erdachte und dann von W. Edward Deming weiterentwickelte Zyklus sieht eine kontinuierliche Abfolge von „Plan-Do-Check-Act“ vor. Einer Planungsphase schließt sich eine Ausführungsphase an. Die Effekte dieser Ausführungen werden dann in einer Überprüfungsphase kontrolliert und in einer Verbesserungsphase weiter optimiert. Damit ist der Kreis jedoch nicht abgeschlossen, sondern im Sinne einer kontinuierlichen Verbesserung schließt sich der letzten Phase die nächste Planungsphase an (vgl. Shewhart 1939). Die vier Phasen sind also ein nie endender Prozess ohne Anfang und Ende. Dieser Kreis sollte, so W. Edward Deming (1986), nicht nur die individuelle Perspektive, sondern die gesamten organisatorischen Prozesse bestimmen. Im Rahmen des Total Quality Managements entsteht daraus dann ein nicht enden wollender Kreis von Interventionen (vgl. Dean/ Bowen 1994).

Auch im Marketing gibt es ähnliche zyklushaft gedachte Konzepte. Das Ziel bestehe hier nicht mehr darin, im Sinne eines transaktionskostenorientierten Marketing die Produkte beim Kunden abladen zu können. Vielmehr komme es im Sinne von Customer-Relation-Management, Marketing von Beziehungen und Kundenloyalitätsmanagement darauf an, die Kunden in langfristige Betreuungsverhältnisse zu integrieren (vgl. Kotler 1984). Vor dem Kaufakt sollte bereits eine intensive Erforschung der Kundenbedürfnisse stattfinden. Auf diese Kundenbedürfnisse ausgerichtet, sollten dann die verschiedenen Marketing-Aktionen geplant und durchgeführt werden. Nach dem Kaufakt sollten die Kunden nach ihrer Zufriedenheit mit dem Produkt und dem Service befragt werden und damit weitere, vielleicht erst durch den Kaufakt geweckte Bedürfnisse identifiziert werden (vgl. Kotler/Keller 2005).

Es spricht vieles dafür, dass sich besonders in den Gewährleistungseinheiten, also den Einheiten, die problematische Umwelteffekte wie Zulieferprobleme (z.B. Abteilung Einkauf), Personalschwankungen (z.B. Abteilung Personal) oder Absatzprobleme (z.B. Abteilung Lager oder Abteilung Verkauf) abfedern und damit einen weitgehend ungestörten Wertschöpfungsprozess sicherstellen (vgl. dazu Thompson 1967, 20 f.), die Ausbildung von Zyklen beobachten lässt. Durch diese Zyklen können die jeweiligen Gewährleistungseinheiten ihre Arbeit auf Dauer stellen und in einem gewissen Rahmen unabhängig gegen "Nachfrageschwankungen" aus den technischen Kernen der Organisation machen. 
Aber die Chancen zur Ausbildung dieser Zyklen scheinen unterschiedlich gut zu sein. Sowohl der Qualitätszyklus als auch der Marketingzyklus sind geschlossene Systeme. Der Übergang von der Durchführungsphase zur Evaluation und dann zur nächsten Planungs- und Durchführungsphase wird in der Regel als weitgehend unproblematischer Prozess betrachtet. Beim Personalentwicklungszyklus ist dieser Prozess jedoch, wie im Folgenden gezeigt wird, deutlich problematischer.

\section{Das Missing Link im Personalentwicklungszyklus: Die Messung der Fortschrittskontrolle}

Die Schwierigkeit beim Personalentwicklungszyklus besteht in der Evaluierung der Maßnahmen, z.B. bei folgenden Fragen: Wie verbessern sich die Leistungen von Führungskräften, die nach Personaldiagnostik entsprechende Personalentwicklungsmaßnahmen durchlaufen haben? Erzielen die Vertriebsmitarbeiter monetär bessere Ergebnisse nach einem Coaching? Für viele Organisationen stellt die Fortschrittsevaluation das fehlende Verbindungsglied im Personalentwicklungszyklus dar. In den letzten fünf Jahrzehnten wurden zwar vielfältige Instrumente zur Evaluierung von Personalentwicklungsmaßnahmen entwickelt, aber diese messen fast ausschließlich in Form von „Happiness-Indexen“ die Zufriedenheit mit einem Seminar, einem Coaching oder einer externen Weiterbildung.

\subsection{Jenseits der Happiness-Indexe}

Dass es jedoch zentrale Unterschiede zwischen der Zufriedenheit unmittelbar nach dem Seminar, dem im Seminar erzielten Wissensfortschritt, den konkreten Verhaltensänderungen und den Effizienz- und Effektivitätseffekten gibt, wurde bereits von Donald L. Kirkpatrick (1959; zuletzt 1998) thematisiert. Die Zufriedenheitseffekte nach dem Seminar (reaction) können mit Feedback-Formularen relativ einfach erhoben werden. Auch eine Evaluation von Wissensfortschritten (learning) kann durch Tests vor und nach einer Maßnahme noch methodisch sauber gesichert festgestellt werden. Änderungen des Verhaltens (behaviour) werden zwar teilweise durch die Managementdiagnostik erhoben, aber gerade bei diesen diagnostischen Maßnahmen herrscht noch große Unsicherheit. Es gibt bisher kaum allgemein akzeptierte Messinstrumente, mit denen die Veränderung von Verhalten festgestellt werden kann. Noch schwieriger gestaltet sich die Mes- 
sung von ökonomischen Veränderungen (results). Zwar gibt es auch hier Instrumente der Unternehmensdiagnostik, aber diese haben sich bisher als Instrumente der Personalentwicklung kaum durchgesetzt.

Obwohl sich viele Unternehmen noch auf die Erstellung eines „HappinessIndex" am Ende einer Maßnahme beschränken (vgl. Ralphs/Stephan 1986), gibt es doch eine deutliche Tendenz, auch die Änderung des Verhaltens und weitergehend die Veränderung der ökonomischen Ergebnisse zu messen. In einem Unternehmen beklagt der Personalentwickler, dass bisher nur "Zufriedenheit" abgefragt wird. „Verhaltensänderung wird nicht gemessen." Hier müsste, so der Personalentwickler, sich das Unternehmen stärker engagieren (Organisation L, Interview 12). Auch in einem anderen Unternehmen propagiert der Leiter der Personalentwicklung, dass wenigstens eine "gewisse Messbarkeit, den Erfolg von Coaching darstellen zu können" möglich sein müsse (Interview 6, Organisation F). In einem weiteren Unternehmen wird bereits mit einem selbst entwickelten Fragebogen die Zufriedenheit mit einem Coaching gemessen. Der Personalentwickler ist mit dieser Selbstevaluation aber nicht zufrieden: „Ich möchte nicht nur die Selbstaussagen des Coachee haben, sondern eigentlich hätte ich auch gerne die Rückmeldung aus dem Unternehmen, was sich durch das Coaching auch verbessert hat". Die "schönste Version" sei, so der Personalentwickler, dass beispielsweise nach einer Coachingphase "gemessen wird, ob das Coaching auch erfolgreich war" (Interview 4, Organisation D). Womit hängt dieses Missing Link im Personalentwicklungszyklus zusammen?

\subsection{Evaluationsschwierigkeiten}

Die Gründe für die Schwierigkeiten bei der Durchführung von Evaluationen sind in der Literatur sehr gut aufgearbeitet; nichtsdestotrotz wird weiter nach geeigneten Evaluationsinstrumenten gesucht. Ein Grund, weswegen Evaluationen in diesem Bereich schwierig sind, ist die Tatsache, dass der Ertrag von Weiterbildungsmaßnahmen sich gerade bei Führungskräften nicht monetär bestimmen lässt (vgl. Grünefeld 1989, S. 187ff.; Neuberger 1991, S. 302). Je komplexer die Tätigkeit einer Führungskraft ist, desto schwieriger wird es, eine konkrete Leistung monetär zu bestimmen. Bei einer Fließbandarbeiterin, also einer Tätigkeit im technischen Kern eines Unternehmens, mag es sehr wohl noch möglich sein, die Effekte von Maßnahmen auch in Dollar oder Euro zu bestimmen. Bei allen konzeptionellen Schwächen und Interpretationskontroversen haben dies bereits die bekannten Experimente in den Hawthorne-Werken gezeigt (vgl. Roethlisberger/ 
Dickson 1939; kritisch dazu z.B. Bramel/Friend 1981; Moldaschl/Weber 1998; Luhmann 2000a, S. 22f.). Aber sobald die Tätigkeiten komplexer werden, wird die monetäre Bemessung einer Leistung zunehmend schwieriger.

Der zweite Grund liegt darin, dass man es bei einer Weiterbildungsmaßnahme mit einer typischen Tätigkeit zu tun hat, deren Effekte sich nicht mit einfachen Ursachen-Wirkungs-Ketten erfassen lassen (vgl. Nork 1989, 50ff.). Die Verbesserung der Leistung eines Managers kann vielfältige Ursachen haben: Vielleicht hat er einige neue Mitarbeiter bekommen, die ihn stimulieren, vielleicht hat sich das Marktumfeld verbessert, oder vielleicht hat er sich auch nur gerade frisch verliebt. Die Zurechnung eines positiven Effekts auf eine bestimmte Personalentwicklungsmaßnahme lässt sich unter diesen Bedingungen kaum vornehmen (vgl. für einen Überblick Baldwin/Ford 1988). Theoretisch könnte man versuchen, diese Zurechnungsprobleme über die Auswahl sehr großer Experimental- und Kontrollgruppen in den Griff zu bekommen, aber hier gibt es bisher kaum nennenswerte Vorlagen (vgl. Künzli 2005, S. 238ff.).

Der dritte Grund liegt in dem Phänomen, was in der Soziologie als Technologiedefizit bezeichnet wird (vgl. Luhmann/Schorr 1979, S. 120). Zwar können Personalentwickler Vorschläge unterbreiten, wie ein Mitarbeiter in einen anderen, für die Organisation und/oder für ihn selbst „besseren“ Zustand überführt werden könnte, aber sie können das Erreichen des anderen Zustandes nicht mit Sicherheit garantieren. Es geht um Personen, die immer auch anders reagieren können, als es sich der Praktiker vorstellt (vgl. Kurtz 2003, S. 98). Man stößt auf das Paradox, dass jemand zielgerichtet verändert werden soll, der sich aber letztlich nur selbst verändern kann (vgl. Horn 1994, S. 204).

In den Organisationen wird die Chance für die Evaluierung der Personalentwicklungsmaßnahmen unterschiedlich eingeschätzt. Während ein überwiegender Teil optimistisch ist, dass sich bei allen methodischen Schwierigkeiten stabile Verfahren entwickeln lassen, zeigen sich einige wenige skeptisch bezüglich der Etablierung dieser Evaluationsinstrumente. Vereinzelt wird auch darauf verwiesen, dass Evaluationen gar nicht gewünscht werden, weil dies „alle Beteiligten unter Druck setzen“ würde, die Frage zu beantworten: „Was hat es denn jetzt gebracht" (Organisation L; Interview 12). 


\section{Der Bedeutungsgewinn der Personalentwicklung in der Organisation: Der Kampf um die personale Deutungshoheit in der Organisation}

Durch den sich ausbildenden Personalentwicklungszyklus, ganz besonders aber durch die Mitsprachemöglichkeiten bei der Personaldiagnostik, scheint die Personalentwicklung einen immer wichtigeren Einfluss auf Personalentscheidungen in der Organisation zu nehmen. Über den Zyklus aus Personaldiagnostik, Intervention und Fortschrittsevaluation gewinnt die Personalentwicklung einen wachsenden Einfluss auf Entscheidungen, die bisher vorrangig im Einflussbereich der Führungskräfte lagen.

\subsection{Die Debatte über Karrierezuständigkeit}

Die Kontroverse, ob die Entscheidung über Karrieren bei den momentanen und zukünftigen Führungskräften oder bei Spezialisten im Personalbereich angesiedelt sein soll, gibt es in Unternehmen, Verwaltungen und anderen Organisationstypen seit über hundert Jahren. Der Karriere-Aspekt spielt deswegen organisatorisch eine wichtige Rolle, weil die Organisationsmitglieder über die Inaussichtstellung von Karrieremöglichkeiten zu einem besonderen Engagement motiviert werden können. Dabei kann die Motivation über den Karrierefaktor sogar in einigen Organisationstypen (z.B. Parteien oder Nichtregierungsorganisationen) höher sein als die Motivation über Bezahlung oder Incentives wie Luxusreisen, edle Essen in Restaurants oder Prostituiertenbesuche (vgl. aus unterschiedlichen Theorieperspektiven z.B. Stinchcombe 1974, S. 130ff.; Türk 1995b, S. 335; Luhmann 2000a, S. 297ff.).

Klassischerweise lag die Entscheidung über die Karrieremöglichkeiten der Mitarbeiter bei den Führungskräften. Diese verfügten damit über eine Machtressource, die viel direkter wirkte als die Möglichkeit, über die Androhung der Entlassung Folgebereitschaft beim Mitarbeiter zu erzielen. Zwar spiele, so Luhmann, die auf Kompetenzen zur dienstlichen Weisung beruhende formale Organisation eine wichtige Rolle, die „faktische Macht" hänge aber weit mehr vom Einfluss auf Karrieren ab. Macht in Organisationen beruhe also nicht so sehr auf der Disposition über Ein- oder Austritt in die Organisation, der so genannten Exitmacht (bei Luhmann Organisationsmacht), sondern vielmehr auf der Disposition über Stellenbesetzungen, also der Karrieremacht (bei Luhmann Personalmacht). Das hänge 
damit zusammen, dass der Mitgliedschaftsentzug viel seltener relevant wird als Entscheidungen über Stellenbesetzungen. Um die Mitgliedschaft zu erhalten, reiche es aus, die Minimalanforderungen zu erfüllen und sich nicht offen gegen Anweisungen des Vorgesetzten aufzulehnen. Um im System Karriere zu machen, sei dagegen viel mehr erforderlich. Wer dies wolle, bekomme die Personalmacht zu spüren (Luhmann 1975, S. 104f.; 2000a, S. 102f. und 280).

Diese Zuordnung geriet jedoch zunehmend in die Kritik. Anknüpfend an die Personalmacht von Vorgesetzten wurde argumentiert, dass - bei einer starken Stellung der Vorgesetzten - die Karriere eines Untergebenen häufig nicht von dessen Leistungsfähigkeit, sondern von dessen Folgebereitschaft abhänge. Bei der Karriereentscheidung durch Führungskräfte könnten in Unternehmen dann auch noch „außerorganisatorische Kriterien“ wie Religion, Ethnizität, politische Einstellungen oder Aktivitäten in anderen Organisationen eine Rolle spielen (vgl. Dalton 1951). In der öffentlichen Verwaltung spielten häufig Ämterpatronage und nicht so sehr die wahrgenommene Leistungsfähigkeit eine Rolle. Karrieren seien häufig von parteipolitischen Betriebsgruppen, Jahrgangsgeflechten oder Fachbruderschaften abhängig (vgl. Bosetzky 1974).

Seit den 1970er Jahren sind die Kompetenzen über die Auswahl von Karrieren - jedenfalls in großen Verwaltungen und Unternehmen - zunehmend auf das Personalwesen übergegangen. Bei der Allianz, so der Bericht eines Personalentwicklers über die Erfahrung aus anderen Unternehmen, habe man „interne Assessment-Center": „Wenn Sie als Führungskraft von der ersten auf die zweite Stufe wollen, dann müssen Sie dort durch, und wenn Sie das zweimal nicht bestanden haben, dann werden Sie das nicht" (Organisation D, Interview 4). In den Assessment-Centern eines der befragten Großunternehmen wird z.B. die "analytische und strategische Kompetenz", die „Leadership-Fähigkeit“, die „Marktund Kundenorientierung", die „Veränderungskompetenz", die „soziale Kompetenz" und die „interkulturelle Kompetenz" von zukünftigen Führungskräften abgefragt. „Ohne Assessment", so einer der Personalentwickler, „,sei eine Karriere nicht möglich" (Organisation G, Interview 7).

In dem Maße, in dem die Personalentscheidungen durchrationalisiert werden und dem Einfluss vieler Personen unterliegen, verliert die Karriere als Machtressource von Vorgesetzten an Bedeutung. Die Manipulation wird für den Vorgesetzten schwierig, weil er einen Mitarbeiter nicht mehr einfach organisationsintern fördern oder blockieren kann. Für den Untergebenen wird es zunehmend undurchsichtig, ob und wie sich ein Wohlverhalten gegenüber den Vorgesetzten auf seine Karriere auswirkt. Zwar könnte der Vorgesetzte vielleicht noch die Bewertung von Untergebenen auf einigen Skalen der Personalentwickler 
kontrollieren, aber es ist zweifelhaft, ob die Vorgesetzten damit überhaupt noch effektiv Personalmacht ausüben können (vgl. Luhmann 1975, S.107).

\subsection{Erklärungen für die Verschiebung der Personalmacht}

Wie konnten die Personaler in diesem Feld gegenüber den General Managern so an Einfluss gewinnen? Eine erste Erklärung liegt darin, dass die Förderung von Mitarbeitern für Führungskräfte nur eine von vielen Aufgaben ist, während dies für die Personalentwicklung zu ihrem zentralen Aufgabenfeld gehört. Dies führt dazu, dass die Karrieresysteme besonders in Unternehmen, Verwaltungen und Krankenhäusern vorrangig auf Betreiben der Personalentwicklung ausgearbeitet wurden. Die Führungskräfte der anderen Bereiche nehmen dabei häufig nur Mitspracherechte in Anspruch - interessiert sie vorrangig nur die Karriere ihrer eigenen Mitarbeiter, nicht jedoch das generalisierte Karrieresystem. Die Konflikte zwischen General Managern und Personalentwicklern treten dann auch nicht vorrangig bei der Diskussion über das System auf, sondern bei der Diskussion über konkrete Personalentscheidungen in den Assessment-Centern. Ein Vorstandsmitglied beklagt sich darüber, dass sein persönlicher Assistent bei dem entsprechenden Auswahlverfahren von den Psychologen als nicht tauglich für die nächste Karrierestufe eingestuft wird. Die Leiterin eines Geschäftsbereichs drückt - gegen eine negative Beurteilung im Assessment-Center - ihren Kandidaten für eine Führungsposition durch.

Eine zweite Erklärung liegt darin, dass mit der Einschaltung der Personalentwicklung in die Karriereplanung und die Nutzung von Personaldiagnostik die Verantwortung für die Personalentscheidung diffundiert. Die in einem Assessment-Center getroffene Entscheidung, einen High-Potential auf eine wichtige Position zu heben, ist nicht mehr auf einen bestimmten Entscheider zurechenbar. Die Entscheidung, einen Mitarbeiter zu entlassen, fällt leichter, wenn dieser vorher durch einen Zyklus aus Personaldiagnostik, Coaching und Messung des Fortschritts getrieben wurde, denn die Entlassungsentscheidung wird durch den Personalentwicklungszyklus "objektiviert" (nicht mehr auf eine konkrete Person zurechenbar).

Diese Tendenz zur Verantwortungsdiffusion kommt dem strategischen Verhalten von Führungskräften in vielen Organisationen entgegen, denn so ist es möglich, die Verantwortung für eine Entscheidung möglichst lange im Unklaren zu lassen (vgl. als beste Fallstudie dazu Jackall 1988, 78ff.). Im Falle des Versagens eines Mitarbeiters auf einer neuen Position kann die Verantwortung auf „das 
Verfahren" geschoben werden. Im Fall des Erfolges kann man sich immer noch nachträglich als „Entdecker“ zu erkennen geben und die damals getroffene Personalentscheidung auf sich zurechnen lassen. Der Erfolg hat bekanntlich viele Väter (und Mütter).

\subsection{Eine neue mikropolitische Situation der Karriere}

Die Ausbildung des Personalentwicklungszyklus hat jedoch nicht dazu geführt, dass die Personaler in den konkreten Karriereentscheidungen das Sagen bekommen haben. In vielen Unternehmen, Verwaltungen und Krankenhäusern ist eine Situation entstanden, in der die Personalentwicklung bei der Durchsetzung des Karrieresystems die Oberhand gewonnen hat, während sich bei den konkreten Personalentscheidungen häufig noch die betroffenen Führungskräfte - auch gegen das offizielle Regelwerk - durchsetzen können. Die Führungskräfte geben in einigen konkreten Fällen die Macht über Personalentscheidungen nicht ab. Ein Vorstandsmitglied teilt vorher indirekt mit, dass ein Scheitern seiner Assistentin beim Assessment-Center nicht geduldet wird. Die Geschäftsführerin eines wichtigen Tochterunternehmens verlangt ein spezielles Coaching für einen Schützling, um diesen erfolgreich durch den Personalentwicklungszyklus zu bekommen.

Der Personalentwickler einer Bank berichtet, dass das „Senioritäts- und Vorstandsprinzip" zwar offiziell abgeschafft wurde, es aber in konkreten Entscheidungssituationen immer wieder durchkomme. Die Personalentwicklung habe ein "aufwändiges Verfahren“ und könne feststellen, dass ein Kandidat nur ein „kleines Gap zum Anforderungsprofil“ habe, ein anderer jedoch "ein größeres“. Wenn dann aber ein Fachbereich sagt, der mit dem "größeren ist leider der einzige Fachmann, der im IT-Bereich das Programm xyz beherrscht“ oder ein "Spezialist für den Finanzsektor ist", dann „wird er trotzdem Führungskraft.“ Dies werde sich erst ändern, wenn das Verfahren von einem "Entwicklungsverfahren“ immer mehr zu einem „Entscheidungsverfahren“ werde (Organisation H, Interview 8). Gerade wenn der Personalentwicklungszyklus in Organisationen noch nicht komplett installiert ist, scheinen diese mikropolitischen Interventionen häufig erfolgreich zu sein. 


\section{$5 \quad$ Neue Dilemmata der Personalentwicklung}

Durch die Ausbildung des Personalentwicklungszyklus hat die Arbeit in der Personalentwicklung einen neuen Charakter bekommen. Schon der Personalbereich wurde häufig als relativ einflussloser Bereich in der Organisation beschrieben. Er hätte lediglich einen Platz auf der „Rückbank“ der Organisation finden können. Die Personaler seien lange Zeit von Vorstandsbereichen ausgeschlossen worden. Sie würden zwischen den Managern und den Arbeitern "feststecken“. Der Personalentwicklung wird dabei häufig eine noch geringere Rolle zugestanden. Weil sie letztlich versuche, so etwas veränderungsresistentes wie die erwachsene Person zu verändern, habe sie lediglich einen schwachen Hebel in der Organisation (vgl. Kühl 2008, Kapitel 6). Der hohe Anteil von Frauen in der Personalentwicklung sei, so das häufig nur hinter vorgehaltener Hand artikulierte Argument, ein deutliches Indiz für die Einflusslosigkeit dieser Abteilung (vgl. für eine Studie über die Geschichte von Personalabteilungen Watson 1977; siehe auch Ferris et al. 1996: 208). Durch die Ausbildung des Personalentwicklungszyklus lässt sich eine Zunahme des Einflusses von Personalentwicklern in der Organisation beobachten. Dies führt aber zu neuen Dilemma-Situationen der Personalentwickler.

\subsection{Das Erwartungsdilemma: Wie befriedigt man gestiegene Erwartungen der Führungskräfte?}

Das erste Dilemma entsteht dadurch, dass durch die Personalbewertung und Personalauswahl die eigene Position in der Organisation gestärkt wird und damit auch eine Professionalisierung der Personalentwicklung begünstigt wird, auf der anderen Seite aber Erwartungen geweckt werden, die in der Regel durch die Personalentwicklung selbst nicht erfüllt werden können.

In Organisationen, in denen die Vorgesetzten über Karrieren entschieden, basierte das Karrieremodell häufig auf den in der Organisation etablierten Seilschaften. Diese Seilschaften ziehen sich häufig über mehrere Hierarchieebenen. Auch wenn dieses Karrierekonzept in der Managementliteratur verpönt ist, so lässt sich doch feststellen, dass die Karriereerwartungen in einer Organisation durch dieses System gut gemanagt werden konnten. Das jeweils eine Hierarchiestufe höher stehende Mitglied einer Seilschaft war Ansprechpartner für die Karrierewünsche der anderen Mitarbeiter und stand unter dem Druck, die anderen Mitarbeiter bei einem eigenen Karrieresprung mitzuziehen. Da diese Seilschaften 
durch die „PE-lerisierung" der Organisation tendenziell geschwächt werden, entfällt damit auch zunehmend die Verantwortung von Führungskräften, „ihre Mitarbeiter" zu versorgen. Die Personalentwicklung sieht sich zunehmend unter Druck, weil den bei Führungskräfte-Audits gut positionierten Mitarbeitern auch bei abgeflachten Hierarchien Entwicklungsperspektiven geboten werden müssen. Die vorrangig von Personalentwicklern vorangetriebene Debatte über „Fachkarrieren“ und „Projektkarrieren“ entstammt ganz maßgeblich diesen Entwicklungen (vgl. Organisation D, Interview 4).

Aber auch diese Suche nach „Fachkarrieren“ und „Projektkarrieren“ kann nicht über das Dilemma der Personalentwicklung hinwegtäuschen. Ihre Stärkung in Fragen der Personalauswahl, Personalbewertung und Personalentwicklung, die nicht zuletzt aus der Schließung des Personalentwicklungszyklus resultiert, korreliert nur sehr eingeschränkt mit einer Zunahme an Möglichkeiten, Mitarbeitern in Organisationen konkrete Perspektiven anbieten zu können. Die Personalentwickler stecken in dem Widerspruch, mit der Schließung des Zyklus ihre eigene Stellung auszubauen, die dadurch geweckten Erwartungen dann aber nicht befriedigen zu können.

\subsection{Das Legitimationsdilemma der Personalentwicklung: Wie weist man den Nutzen von Personalentwicklungsmaßnahmen nach?}

Das zweite Dilemma bezieht sich auf den Nutzennachweis. Auf der einen Seite nimmt der Druck, den Nutzen von Personalentwicklungsmaßnahmen monetär nachweisen zu können, immer mehr zu. Je stärker die Mitarbeiter durch den Personalentwicklungszyklus in eine Rundumbetreuung geraten, desto größer ist die Erwartung seitens der Organisationsspitze, dass die Ausgaben gerechtfertigt sind. Auf der anderen Seite spricht wenig dafür, dass sich die Tätigkeiten in Organisationen - und erst recht nicht die Funktionsweise der einzelnen Personen so stark vereinfachen lassen, dass sich eine entsprechende Berechnung vornehmen ließe.

Der Personalentwickler eines Großkonzerns berichtet mit einem Lachen von "Amerikanern", die für Coaching ein Return on Investment von 529\% berechnet haben, und bringt dann zum Ausdruck, dass einen allein schon die präzise Zahl „529\%“ stutzig machen muss. ${ }^{3}$ Aber es sei nötig, so der Personalentwickler, eine

\footnotetext{
3 Andreas Taffertshofer (2007) kommentiert die auch in Publikationen postulierten Return-onInvestment-Werte für Personalentwicklungsmaßnahmen von 545\% (McGovern 2001) oder 788\% (Ander-
} 
"gewisse Messbarkeit" des Coachingerfolgs zu entwickeln. Gerade in der Diskussion mit Ingenieuren sei es hilfreich, wenn man den Nutzen in Form von Zahlen nachweisen könne. In der Unternehmenskultur sei es immer besser, „man zeigt eine Zahl, als dass man sagt, jetzt guck mal, es gibt so viele zufriedene Leute" (Organisation F, Interview 6).

So schwanken viele Personalentwickler zwischen zwei Strategien hin und her: der Verweigerung eines konkreten Nutzennachweises und der damit einhergehenden Inkaufnahme des Legitimitätsproblems sowie der Übernahme simplifizierender Berechnungsmethoden, deren Grundlagen man eigentlich aus professionellen Überlegungen ablehnen müsste. Gerade bei der letzten Strategie bildet sich eine Form von organisationaler Heuchelei aus, die vielleicht das Legitimationsproblem zu lösen hilft, aber interne Lernprozesse in der Organisation blockieren kann; denn es besteht immer die Gefahr, dass einzelne Personalentwickler die produzierten Zahlen ernst nehmen und zum Anlass für Lernprozesse nehmen (vgl. zum Konzept der Heuchelei Brunsson 1989).

\subsection{Das Reputationsdilemma: Zwischen Innen- und Außenorientierung in der Organisation}

Die Ausbildung des Personalentwicklungszyklus führt zu einer stärkeren Außenorientierung der Personalentwicklung. Gerade an der Debatte über die Personaldiagnostikmaßnahmen und die Instrumente zur Nutzenmessung erkennt man, dass sich Personalentwickler an den Standards in der Personalentwicklerszene orientieren und selbst versuchen, die entwickelten Instrumente und gemachten Erfahrungen zurückzuspielen. Gerade dadurch bauen sich "Stars“ in der Szene auf.

Das Problem ist jedoch, dass diese Reputation nicht in die Organisation zurückübersetzt werden kann. Eine Personalentwicklerin mag in der Szene der PEler eine Koryphäe sein und sich durch Vorträge, Artikel und Ausbildungen profilieren. Dies trägt aber kaum zur Stärkung ihrer eigenen Position in der Organisation bei. Der Personalentwickler eines Unternehmens erklärte, dass sein Bereich in der Szene ein „Renommee“ aufgebaut habe, aber für ein Unternehmen, das Autos baut, sei es „relativ wurscht, welches Renommee ein indirekter Bereich

son 2001) mit der sarkastischen Frage, weswegen nicht alle Unternehmen angesichts dieser Effizienzmöglichkeiten sich einfach auf Coaching konzentrieren würden. 
hat". Wenn man "Geld sparen kann, dann macht man ihn klein“ (Organisation F, Interview 6).

Dieses Schisma zwischen interner und externer Reputation ist spezifisch für Organisationsmitglieder, die in den indirekten Bereichen einer Organisation tätig sind. Das Problem erklärt sich damit, dass die Zwecksetzung für Personalentwickler nicht mit der Zwecksetzung der Gesamtorganisation korreliert. Während der Zweck eines Unternehmens z.B. der Verkauf vieler Autos ist, besteht die Zwecksetzung der Personalentwickler darin, bei der Einstellung möglichst fähiger Manager Unterstützung zu leisten. Selbstverständlich präsentiert sich der Zweck der Personalentwicklung als ein Mittel zur Erreichung des Oberzwecks der Organisation, aber der Unterzweck ist nicht als "Schauseite der Organisation" (vgl. Luhmann 1964, S. 112) präsentierbar.

Der Unterschied wird bei einer Betrachtung von Professionals in den unmittelbaren Wertschöpfungsbereichen einer Organisation deutlich: In einem Krankenhaus korreliert die Reputation eines Arztes außerhalb der Klinik in der Regel mit seiner Stellung innerhalb der Organisation. Ein Ingenieur, der außerhalb eines Automobilunternehmens als Star gilt, kann diese Reputation direkt in organisationsinterne Reputation ummünzen. Es ist das Schicksal des Personals in den indirekten Bereichen, dass es diese Übersetzungsmöglichkeit von externer in interne Reputation nicht hat. Personalentwickler bewegen sich deswegen in einem Spannungsfeld zwischen Innen- und Außenorientierung. Zwar wird es häufig so dargestellt, dass die Außenorientierung auch dem Know-how-Aufbau in der Organisation dient, in der Realität sind dies aber häufig widersprüchliche Anforderungen, die miteinander vereinbart werden müssen.

\section{Schlussbemerkung: Zur Einschätzung des Personalentwicklungszyklus}

Die These vom Personalentwicklungszyklus dient hier erst einmal als ein Interpretationsangebot, das aus dem analysierten empirischen Material entwickelt wurde. Sie bedarf jedoch unseres Erachtens einer weiteren Überprüfung. $\mathrm{Zu}$ dieser Überprüfung reicht eine direkte quantitative Befragung von Personalentwicklern jedoch nicht aus, weil auf die Frage nach dem Grund des Booms von personalentwicklerischen Interventionsinstrumenten "legitime“ Antworten zu erwarten sind. Bei der direkten Frage sind, wie die unzähligen Studien von Beratungsfirmen zeigen, eher Antworten zu erwarten, die die Gründe entweder in abstrakten gesellschaftlichen Veränderungen wie "Globalisierung“, ",gestiegenem 
Wettbewerbsdruck“ oder "neuen Technologien“ sehen oder eher die personenorientierten Erklärungen wie „neue Aufgabe“, „höhere Führungsposition“, „Reorganisation“ oder „interner Konflikt“ wiedergeben.

In einem nächsten Schritt käme es darauf an, den Zusammenhang von Managementdiagnostik, personalentwicklerischen Interventionen und Fortschrittsevaluation noch stärker in einem organisatorischen Kontext zu interpretieren. Dafür könnte es z.B. hilfreich sein, systematisch solche „Karrierefälle“ oder „Stellenbesetzungsprozeduren" $\mathrm{zu}$ untersuchen, in denen Linienmanagement und Personalentwicklung grundlegend unterschiedlicher Auffassung gewesen sind. Über einen solchen Entwicklungsfokus könnte deutlicher herausgearbeitet werden, ob, wie vermutet, eine Schließung des Personalentwicklungszyklus die Position der Personalentwickler bei diesen zentralen Karriereentscheidungen stützt.

Die Ausbildung des Personalentwicklungszyklus ist im Zusammenhang mit der Neupositionierung der Personalfunktion im Allgemeinen und der Personalentwicklung im Speziellen in den letzten Jahren zu sehen. Während in den 1980er Jahren die Debatte vorrangig über eine Professionalisierung im Personalbereich geführt wurde (vgl. Wächter 1987), hat sich die Debatte seit den 1990er Jahren immer stärker auf die Frage verlagert, wie der Personalbereich und ganz besonders die Personalentwicklung ihre Leistung gegenüber ihren internen Kunden zu erbringen hat. Dabei setzte sich in vielen Organisationen das Leitbild durch, dass sich gerade die Personalentwicklung als Profitcenter im Unternehmen zu bewähren hat. ${ }^{4}$ Die Personalentwickler sollten, so die Idee, als „Unternehmer im Unternehmen" ihre Leistungen anderen Bereichen im Unternehmen gegen Berechnung anbieten (vgl. Luo 2006). Hier spiegelt sich eine Tendenz wider, die auch bei anderen Gewährleistungseinheiten in Unternehmen zu beobachten ist. Bei der Logistik, beim Werkschutz oder bei der Pressearbeit werden Überlegungen angestellt, die Gewährleistungsarbeiten nicht mehr als reine Kostenfunktion erbringen zu lassen, sondern die Gewährleistungseinheiten als Profitcenter in Wettbewerb zu anderen Anbietern treten zu lassen.

Auch wenn es sich bei diesen Modellen vielfach nur um Leitbilder handelt, an denen man sich zu orientieren sucht, ${ }^{5}$ stellt man doch fest, dass dies auch die Ausrichtung der Organisation verändert. Der Personalentwicklungszyklus lässt sich als Komplettangebot in die verselbstständigte Personalentwicklung integrie-

\footnotetext{
${ }^{4}$ Gerade in den größeren Organisationen in dem Untersuchungssample lässt sich dieser Trend beobachten (siehe z.B. Organisation 6).

${ }^{5}$ Es fehlen bisher quantitative Untersuchungen darüber, wie verbreitet die Profitcenter-Idee im Personalentwicklungsbereich ist und welches der Modelle sich durchsetzt.
} 
ren und führt dazu, dass - unabhängig von der Personalsituation der Organisation - immer eine personalentwicklerische Handlungsnotwendigkeit besteht.

\section{Literatur}

Anderson, Merrill C. (2001): Case Study on the Return on Investment of Executive Coaching. Online: http://www.coachcentral.info/Merrill_study.htm.

Backhausen, Wilhelm; Jean-Paul Thommen (2004): Durch systemisches Denken zu innovativer Personalentwicklung. 2. akt. Aufl. Wiesbaden: Gabler.

Baldwin, Timothy T.; Kevin J. Ford (1988): Transfer of Training: A Review and Directions for Future Research. In: Personnel Psychology, Jg. 41, S. 63-105.

Beck, Ulrich (1986): Risikogesellschaft. Auf dem Weg in eine andere Moderne. Frankfurt am Main: Suhrkamp.

Belardi, Nando (2002): Supervision. Grundlagen, Techniken, Perspektiven. München: Beck.

Bosetzky, Horst (1974): "Dunkelfaktoren" bei Beförderungen im öffentlichen Dienst. In: Die Verwaltung, Jg. 7, S. 427-438.

Bramel, Dana; Ronald Friend (1981): Hawthorne, the Myth of the Docile Worker, and Class Bias in Psychology. In: American Psychologist, Jg. 36, S. 867-878.

Buchinger, Kurt (2002): Supervision in Wirtschaftsorganisationen. In: Supervision, H. 2/2002, S. 47- 54 .

Dalton, Melville (1951): Informal Factors in Career Achievement. In: American Journal of Sociology, Jg. 56, S. 407-415.

Dean, James W.; David E. Bowen (1994): Management Theory and Total Quality. Improving Research and Practice Through Theory Development. In: Academy of Management Review, Jg. 19, S. 392-418.

Deming, W. Edward (1986): Out of the Crisis. Quality, Productivity, and Competetive Position. Cambridge, Mass.: Massachusetts Institute of Technology.

Ferris, Gerald R. et al. (1996): The Paradox of Diversity in Organizations. In: Gutschelhofer, Alfred; Josef Scheff (Hg.): Paradoxe Management. Widersprüche im Management Management der Widersprüche. Wien: Linde, S. 203-229.

Grünefeld, H.G. (1989): Weiterbildungsaufwand. In: Personalführung, H. 2/1989, S. 1831991.

Horn, Elke (1994): Soziologische Systemtheorie und systemische Familientherapie: Einige Anmerkungen zu den Möglichkeiten eines Dialogs. In: Herlth, Alois et al. (Hg.): Abschied von der Normalfamilie? Partnerschaft contra Elternschaft. Berlin et al.: Springer, S. 203-212.

Jackall, Robert (1988): Moral Mazes. The World of Corporate Managers. New York; Oxford: Oxford University Press.

Kieser, Alfred (1995): Der Situative Ansatz. In: Kieser, Alfred (Hg.): Organisationstheorien. 2. Aufl. Stuttgart; Köln; Berlin: Kohlhammer, S. 155-184. 
Kirkpatrick, Donald L. (1959): Techniques for Evaluation Training Programs. In: Journal of American Society for Training Directors, Jg. 13, S. 28-32.

Kirkpatrick, Donald L. (1998): Evaluating Training Programs: The Four Levels. 2. Aufl. San Francisco: Berrett- Koehler

Kotler, Philip (1984): Marketing Essentials. Englewood Cliffs, NJ: Prentice-Hall.

Kotler, Philip; Kevin Lane Keller (2005): Marketing Management. 12. Aufl. Englewood Cliffd, NJ: Prentice Hall.

Kurtz, Thomas (2003): Gesellschaft, Funktionssystem, Person: Überlegungen zum Bedeutungswandel professioneller Leistung. In: Harald Mieg; Michaela Pfadenhauer (Hg.): Professionelle Leistung - Professional Performance. Positionen zur Professionssoziologie, Konstanz: UVK, S. 89-110.

König, Eckard, Gerda Volmer (2003): Systematisches Coaching. Handbuch für Führungskräfte, Berater und Trainer. 2. unv. Auflage. Weinheim, Basel: Beltz Verlag.

Kühl, Stefan (2000): Das Regenmacher-Phänomen. Widersprüche und Aberglaube im Konzept der lernenden Organisation. Frankfurt a.M.; New York: Campus.

Künzli, Hansjörg (2005): Wirksamkeitsforschung im Führungskräfte-Coaching. In: Organisationsberatung - Supervision - Coaching, Jg. 12, S. 231-243.

Liebold, Renate; Rainer Trinczek (2002): Experteninterview. In: Kühl, Stefan; Petra Strodtholz (Hg.): Methoden der Organisationsforschung. Ein Handbuch. Reinbek: Rowohl, S. 33-70.

Luhmann, Niklas (1964): Funktionen und Folgen formaler Organisation. Berlin: Duncker \& Humblot.

Luhmann, Niklas (1975): Macht. Stuttgart: Enke.

Luhmann, Niklas (2000): Organisation und Entscheidung. Opladen: WDV.

Luhmann, Niklas; Karl-Eberhard Schorr (1979): Das Technologiedefizit der Erziehung und die Pädagogik. In: Zeitschrift für Pädagogik, Jg. 25, S. 345-365.

Luo, Xiaowei (2006): The Spread of a "Human Resources" Culture: Institutional Individualism and the Rise of Personal Development Training. In: Drori, Gili S.; John W. Meyer; Hokyu Hwang (Hg.): Drori, Gili S., John W. Meyer and Hokyu Hwang (Hg) Globalization and Organization: World Society and Organizational Change. Oxford; New York: Oxford University Press.

Luthans, Fred; Suzanne J. Peterson (2003): 360-degree Feedback with Systematic Coaching: Empirical analysis Suggests a Winning Combination. In: Human Resource Management, Jg. 42, S. 243-256.

McGovern, Joy et al. (2001): Maximising the Impact of Executive Coaching: Behavioral Change, Organizational Outcomes, and Return on Investment. In: The Manchester Review, Jg. 6, S. 1-9.

Moldaschl, Manfred (2005): Reflexive Beratung - ein Geschäftsmodell? In: Mohe, Michael (Hg.): Innovative Beratungskonzepte. Ansätze, Fallbeispiele, Reflexionen. Leonberg: Rosenberg, S. 43-68.

Moldaschl, Manfred; Wolfgang G. Weber (1998): The "Three Waves" of Industrial Group Work. Historical Reflections on Current Research on Group Work. In: Human Relations, Jg. 51, S. 347-388. 
Neuberger, Oswald (1991): Personalentwicklung. Stuttgart: Enke.

Nork, Manuela (1989): Management Training. Evaluation, Probleme, Lösungsansätze. München; Mering: Hampp.

Pongratz, Hans J. (2004): Der Typus "Arbeitskraftunternehmer" und sein Reflexionsbedarf. In: Buer, Ferdinand; Gertrud Siller (Hg.): Die flexible Supervision. Herausforderungen - Konzepte - Perspektiven. Wiesbaden: VS-Verlag, S. 17-34.

Ralphs, Lenny T.; Eric Stephan (1986): HRD in the Fortune 500. In: Training and Development Journal, Jg. 40, S. 69-76.

Reichel, René, Reinhold Rabenstein (2001): Kreativ beraten. Methoden, Modelle, Strategien für Beratung, Coaching und Supervision. Münster: Ökotopia Verlag.

Roethlisberger, Fritz Jules; William J. Dickson (1939): Management and the Worker. An Account of a Research Program Conducted by the Western Electric Company, Hawthorne Works, Chicago. Cambridge, Mass.: Harvard University Press.

Shewhart Walter A. (1939): Statistical Method from the Viewpoint of Quality Control. Lancaster, Pa.: Lancaster Press.

Smither, James W. et al. (2003): Can Working with an Executive Coach Improve Multisource Feedback Ratings over Time? A Quasi-experimental Field Study. In: Personnel Psychology, Jg. 56, S. 23-44.

Stinchcombe, Arthur L. (1974): Creating Efficient Industrial Administrations. New York; London: Academic Press.

Strodtholz, Petra; Stefan Kühl (2002): Qualitative Methoden der Organisationsforschung ein Überblick. In: Kühl, Stefan; Petra Strodtholz (Hg.): Methoden der Organisationsforschung. Ein Handbuch. Reinbek bei Hamburg: Rowohlt, S. 11-32.

Taffertshofer, Andreas (2006): Coaching in Arbeitsorganisationen. Zu Mode und Praxis einer persönlichen Beratungsmethode. München: Diplomarbeit am Institut für Soziologie der Uni München.

Thommen, Jean-Paul (2005): Coaching: Modewort oder modernes Managementinstrument. In: Zeitschrift Führung und Organisation, H. 2/2006, S. 64-70.

Thompson, James D. (1967): Organizations in Action. New York et al.: McGraw-Hill.

Türk, Klaus (1995): Entpersonalisierte Führung. In: Kieser, Alfred; Gerhard Reber; Rolf Wunderer (Hg.): Handwörterbuch der Führung. 2. erw. Aufl. Stuttgart: Schäffer-Poeschel, S. 328-340.

Voß, G. Günter; Hans J. Pongratz (1998): Der Arbeitskraftunternehmer. Eine neue Grundform der Ware Arbeitskraft? In: Kölner Zeitschrift für Soziologie und Sozialpsychologie, Jg. 50, S. 131-158.

Watson, Tony J. (1977): The Personnel Managers: A Study in the Sociology of Work and Employment. London: Routledge \& Kegan Paul.

Wächter, Hartmut (1987): Professionalisierung im Personalbereich. In: Die Betriebswirtschaft, Jg. 47, S. 141-150. 